\title{
Non-Friedmann cosmology for the Local Universe, significance of the universal Hubble constant, and short-distance indicators of dark energy
}

\author{
A. D. Chernin ${ }^{1,2,3}$, P. Teerikorpi ${ }^{1}$, and Yu. V. Baryshev ${ }^{4,5}$ \\ 1 Tuorla Observatory, University of Turku, 21500 Piikkiö, Finland \\ 2 Sternberg Astronomical Institute, Moscow University, 119899, Moscow, Russia \\ 3 Division of Astronomy, University of Oulu, 90014, Finland \\ 4 Institute of Astronomy, St.Petersburg State University, Staryj Peterhoff, 198504, St. Petersburg, Russia \\ 5 Isaac Newton Institute of Chile, Saint-Petersburg Branch, Russia
}

Received 8 December 2005 / Accepted 19 April 2006

\begin{abstract}
Based on the increasing evidence of the cosmological relevance of the local Hubble flow, we consider a simple analytical cosmological model for the Local Universe. This is a non-Friedmann model with a non-uniform static space-time. The major dynamical factor controlling the local expansion is the antigravity produced by the omnipresent and permanent dark energy of the cosmic vacuum (or the cosmological constant). The antigravity dominates at larger distances than 1-2 Mpc from the center of the Local group. The model gives a natural explanation of the two key quantitative characteristics of the local expansion flow, which are the local Hubble constant and the velocity dispersion of the flow. The observed kinematical similarity of the local and global flows of expansion is clarified by the model. We analytically demonstrate the efficiency of the vacuum cooling mechanism that allows one to see the Hubble law this close to the Local group. The "universal Hubble constant" $H_{\mathrm{V}}\left(\approx 60 \mathrm{~km} \mathrm{~s}^{-1} \mathrm{Mpc}^{-1}\right)$, depending only on the vacuum density, has special significance locally and globally. The model makes a number of verifiable predictions. It also unexpectedly shows that the dwarf galaxies of the local flow with the shortest distances and lowest redshifts may be the most sensitive indicators of dark energy in our neighborhood.
\end{abstract}

Key words. cosmology: dark matter - cosmology: cosmological parameters - galaxies: Local Group

\section{Introduction}

Cosmology has changed dramatically with the discovery of the cosmic acceleration and dark energy of the cosmic vacuum (or the cosmological constant) from observations of high-redshift supernovae (Riess et al. 1998; Perlmutter et al. 1999). A new "concordance cosmological model" has emerged that describes the accelerating cosmological expansion on cosmological scales beyond the cell of uniformity $(>300-1000 \mathrm{Mpc})$. The model is in good agreement with all cosmological data, including the WMAP results on the CMB anisotropy (Spergel et al. 2003). The Friedmann theory with a non-zero positive cosmological constant serves as a reliable basis for the concordance model, the present-day standard model in cosmology (see e.g. reviews by Chernin 2001; Peebles \& Ratra 2003).

A new field of studies, in which the cosmic vacuum has proved to be important, lies deep inside the cell of uniformity. Cosmological expansion was originally discovered in this Local Volume (distances $<10-20 \mathrm{Mpc}$ ). Our understanding of the physical conditions there has improved so much that, for the first time, we have reliable grounds for finding a solution to the Hubble-Sandage paradox. The paradox lies in the fact that cosmological expansion with a regular linear velocity-distance relation was discovered by Edwin Hubble where it should not be. Indeed, the Friedmann theory predicts and describes the expansion of a smooth, homogeneous self-gravitating medium. But locally, the matter is far from uniform and instead reveals a fairly chaotic spatial distribution of galaxies. Therefore the Friedmann theory cannot be applied to the Local Volume. The paradox was splendidly realized by Allan Sandage (Sandage et al. 1972; Sandage 1986, 1999). He saw a most striking mystery in the fact that the local expansion proceeds in quite a regular way: the linear velocity-distance relation (the Hubble law) with a low velocity dispersion is clearly observed here. Moreover, the local rate of expansion is similar, if not identical, to the global rate characterized by the Hubble constant.

Recently, Thim et al. (2003) have emphasized a related problem: there is no evidence of the expected turn-down of the velocity-distance relation, when one approaches the Local Group (cf. Ekholm et al. 2001). Whiting (2005) notes the similar absence of infall flow around local galaxy groups in general.

Soon after the discovery of the cosmic vacuum, we suggested (Chernin et al. 2000 - hereafter Paper I) that the cosmic vacuum with its omnipresent and perfectly uniform density might provide the dynamical background for a regular quiescent expansion in the Local Volume. Our key argument comes from the fact that the vacuum antigravity dominates the matter gravity of the Local Group at distances larger than 1-2 Mpc from the center of the group (Chernin 2001; Baryshev et al. 2001; Karachentsev et al. 2003). The increasing observational evidence and theoretical arguments for this view have been recently discussed by Teerikorpi et al. (2005). In that paper it was also shown that dark 
energy may explain yet another problem in the Local Volume: why peculiar velocities do not depend on luminosity (mass) (Karachentsev \& Makarov 1996; Whiting 2003, 2005).

This new approach has extended cosmology into the deep interior of the cosmic cell of uniformity. Especially, the Local Volume must be regarded as the nearest cosmologically relevant object, so we find the term "the Local Universe" useful for our relatively small "Local Volume" sample of the Universe which, however, is fairly typical of galaxy space in general. We also speak about "local cosmology", assuming at the same time that the Universe as a whole is described by the concordance model of the traditional "global" cosmology.

In the present paper, we develop our approach further and study a simple analytical non-Friedmann model for the Local Universe (Sects. 2, 3). The model naturally explains two key characteristics of the local expansion flow: the local Hubble constant and the velocity dispersion (Sect. 4). The special significance of the universal "vacuum" Hubble constant is emphasized (Sect. 5). The model predicts that the cosmic antigravity and vacuum density can be detected and measured in our nearest neighborhood (Sect. 6). In Sect. 7, complementary computer simulations are briefly reviewed and a general discussion of the results is given. We summarize the conclusions in Sect. 8 .

\section{Local cosmology: Newtonian theory}

It is clear from the considerations of Sect. 1 that a nonFriedmann counterpart of Friedmann's cosmological model is needed for the Local Universe where the matter distribution is highly non-uniform. In constructing a non-Friedmann theory, we focus first on the nearest and most important distances, 1-5 Mpc, where the observational data are fairly complete and distance determinations are accurate enough (Karachentsev \& Makarov 2001; Karachentsev et al. 2002, 2003a; Thim et al. 2003; Karachentsev et al. 2004; Paturel \& Teerikorpi 2005; Rekola et al. 2005). We start with a simple version of the theory in which the following physical conditions are assumed for the present epoch of cosmic evolution:

1. The environment of the Local Universe (i.e. the distribution and motion of matter outside it) does not affect the dynamics inside it essentially, so one may neglect the matter distribution and motions at distances larger than, say, $5 \mathrm{Mpc}$, in the first and main approximation.

2. The matter mass of the Local Universe is collected almost entirely - with a few percent accuracy - within the area of the Local Group at the distances $\leq 1 \mathrm{Mpc}$ from the barycenter of the group, in this approximation.

3. The gravity field produced by the Local Group is spherically symmetrical and static at the distances $\geq 1.5-2 \mathrm{Mpc}$ from the group barycenter (see below).

4. The interaction of the outflowing dwarf galaxies with each other is negligible.

5. The flow consists of the galaxies that have escaped the gravitational potential well of the Local Group.

\subsection{The equation of motion}

Under these simplified assumptions, but ones that are sufficiently reasonable for the present discussion, the dynamics at distances $1-5 \mathrm{Mpc}$ from us is reduced to the problem of a test particle motion in the gravity field of the Local Group treated as a point-like mass on the background of the antigraviting vacuum. The gravity of the Local Group produces the radial force $-G M / r^{2}$, where $r$ is the distance from the group barycenter and $M=1.5 \times 10^{12} M_{\odot}$ is the group mass tentatively adopted here for calculating certain numerical values (we return to this in Sect. 6.2). The antigravity of the vacuum produces the radial force $G 2 \rho_{\mathrm{V}}\left(\frac{4 \pi}{3} r^{3}\right) / r^{2}$, where $-2 \rho_{\mathrm{V}}=\rho_{\mathrm{V}}+3 p_{\mathrm{V}}$ is the effective gravitating density of vacuum, according to General Relativity (GR) (see e.g. Chernin 2001), and $\rho_{\mathrm{V}}=7 \times 10^{-30} \mathrm{~g} / \mathrm{cm}^{3}$ is the concordance value of the vacuum density.

The expression for the antigravity force is given here in terms of Newtonian mechanics. Its structure looks natural, but it actually needs justification from General Relativity. A static GR space-time for a mass embedded in the uniform vacuum (Sect. 3) gives such a justification; see especially Eq. (14) and compare it with Eq. (4) below.

The radial component of motion in this gravity/antigravity force field obeys the Newtonian equation

$\ddot{r}(t, \chi)=-G M / r^{2}+r / A^{2}$,

where $r(t, \chi)$ is the distance of a particle to the barycenter of the Local group and $\chi$ is the Lagrangian coordinate of the particle. The constant

$A=\left(\frac{8 \pi G}{3} \rho_{\mathrm{V}}\right)^{-1 / 2} \simeq 5 \times 10^{17} \mathrm{~s} \simeq 1.5 \times 10^{28} \mathrm{~cm}$

is the characteristic vacuum time/length; hereafter the speed of light $c=1$ is used in the formulas. It is interesting that the constant vacuum time/length is numerically near the current cosmic age or the distance to the cosmological horizon: $t_{0} \sim A$. A discussion of this fact may be found, for instance, in Chernin (2005).

It is seen from Eq. (1) that the gravity force $\left(\propto 1 / r^{2}\right)$ dominates the antigravity force $(\propto r)$ at smaller distances, and the acceleration is negative there. At the distance

$R_{\mathrm{V}}=\left(G M A^{2}\right)^{1 / 3}=\left(\frac{3}{8 \pi} M / \rho_{\mathrm{V}}\right)^{1 / 3} \simeq 1.3 \mathrm{Mpc}$,

the gravity and antigravity balance each other, so the acceleration is zero at the "zero-gravity sphere" of the radius $R_{\mathrm{V}}$ (Paper I). At larger distances, $r>R_{\mathrm{V}}$, antigravity dominates, and the acceleration is positive.

We mostly study the region outside the zero-gravity sphere where the gravity field is assumed to be static and spherically symmetrical (see above). In fact, computer simulations of the Local group and its gravity (Dolgachev et al. 2003; Chernin et al. 2004) show that the zero-gravity surface is fairly spherically symmetrical at the present epoch and is approximately (with a 10-15\% accuracy, at least) unchanged in size and shape over more than 12 Gyr of the history of the Local Group.

The first integral of Eq. (1) expresses, as usual, the mechanical energy conservation:

$\frac{1}{2} \dot{r}^{2}=G M / r+\frac{1}{2}(r / A)^{2}+\bar{E}$,

where $\bar{E}(\chi)$ is the total mechanical energy of a particle with the Lagrangian coordinate $\chi$ (per its unit mass). Here the potential energy

$U(r)=-G M / r-\frac{1}{2}(r / A)^{2}$

is negative, and both matter and vacuum give to $U$ contributions of the same sign (contrary to the right-hand side of Eq. (1)). 
Because of the vacuum, the gravitational potential cannot be normalized to $U=0$ at $R=\infty$, and $U(R)$ goes to $-\infty$ in both limits of $R \rightarrow 0$ and $R \rightarrow \infty$, which is not a standard situation in mechanics. It means, in particular, that the trajectories with $E<0$ are not necessarily finite. Such behavior of the potential has a clear analogue in General Relativity applied to the same problem (Sect. 3).

\subsection{Radial trajectories}

The dwarf galaxies of the local expansion flow move along approximately radial trajectories in the potential $U(r)$. The total energy of each particle that has escaped from the gravity potential well of the Local Group exceeds the maximal value of the potential $U$ :

$E>U_{\max }=-\frac{3}{2} G M / R_{\mathrm{V}}$.

Asymptotically, when $r$ goes to infinity and the vacuum dominates entirely, the solution of Eq. (4) is exponential,

$r(t, \chi) \propto \exp (t / A)$

so that the linear velocity-distance law, $\dot{r}=r / A_{\mathrm{V}}$, appears with the constant expansion rate:

$H_{\mathrm{V}} \equiv 1 / A \simeq 60 \mathrm{~km} \mathrm{~s}^{-1} \mathrm{Mpc}^{-1}$.

The quantity $H_{\mathrm{V}}$ is a universal physical constant directly related to the vacuum density (or the cosmological constant); its provisional numerical value, adopted here, comes from the concordance data on $\rho_{\mathrm{V}}$. We call $H_{\mathrm{V}}$ the universal Hubble constant.

Generally, by integrating Eq. (4), one gets:

$t-T(\chi)=\int\left[2 G M / r+(r / A)^{2}+2 \bar{E}\right]^{-1 / 2} \mathrm{~d} r$,

where $T(\chi)$ is the second integration constant. Two timeindependent functions of the Lagrangian coordinates, $\bar{E}(\chi)$ and $T(\chi)$, are determined by the particle's "initial conditions" that are its velocity and distance from the center at a given moment of time.

In the simplest case of the parabolic $(\bar{E}=0)$ motion, the exact integral of Eq. (9) is expressed in terms of elementary functions:

$r(t, \chi) \propto[\sinh (3 t / 2 A)]^{2 / 3}$.

\subsection{Local vs. global}

It is significant that the mathematical structure of Eq. (4) is similar to that of the Friedmann cosmological equation for the global Friedmann radius $R(t)$ :

$$
\frac{1}{2} \dot{R}(t, \chi)^{2}=G M(\chi) / R+\frac{1}{2} R^{2} / A^{2}+E(\chi),
$$

where $M(\chi)$ is the time-independent mass of the non-relativistic matter within the sphere of the radius $R(\chi, t)$, and $\chi$ is the Lagrangian coordinate that corresponds to the Eulerian coordinate $R(t, \chi)$; the time-independent value $E(\chi)=k(R / a)^{2}, a$ is the curvature radius, $-k=-1,0,+1$ is the sign of the $3 \mathrm{D}$ space curvature. As is well-known, the function $R(t, \chi)$ is a product of the curvature radius $a(t)$ and a function of the Lagrangian coordinate. In terms of physics, the difference of Eq. (11) from Eq. (4) is in the dependence of the mass $M(\chi)$ on the Lagrangian coordinate in the Friedmann equation, due to the uniform matter distribution. According to the non-Friedmann Eq. (4), each body moves in the potential of the same gravitational mass. Clearly, the solution of Eq. (11) has the same mathematical form as Eq. (10) for the parabolic $(E=0)$ motion.

It is especially suggestive that, in the limit of large $R$ and large $r$, both equations lead to the same exponential solution with the linear velocity-distance relation and the universal Hubble constant $H_{\mathrm{V}}=1 / A$. This similarity demonstrates that both local and global expansion flows are controlled by the same physical factor, the cosmic vacuum with its perfectly uniform density, when the vacuum entirely dominates. In this limit, the global cosmological expansion and the local Hubble flow are parabolic. The only constant physical parameter for both is the universal Hubble constant in this asymptotic regime.

It is also remarkable that the real Local Universe and the global Universe are now both rather close to this common limit. Thanks to this underlying physical cause, the observed values of $H_{\text {local }}$ and $H_{0}$ are so close to each other because each of them is close to $H_{\mathrm{V}}$.

Equations (1)-(10) constitute the basic equations of our non-Friedmann model for the Local Universe. To describe the present-day local flow, we can put $t=t_{0} \simeq 14 \mathrm{Gyr}$ in Eqs. (1)-(10). Then we have the dependence of the acceleration and velocity of the flow particles on the distance $r$ at the present epoch. Especially informative is the phase (velocity-distance) relation given by Eqs. (4) and (10), showing that the velocity of a particle is determined by its distance and energy $E$, while the distance itself depends on both energy and the "initial moment" $T$ that are different for different particles (e.g. particles emerge at different times from within the zero-gravity surface). This set of equations in combination with observational data gives a clear picture of the evolution and the present kinematics of the expansion flow in the gravity/antigravity force field of the Local Universe. They also clarify the major trends in the evolution of the flow during the whole lifetime $(\simeq 12-13 \mathrm{Gyr})$ of the Local group (see below).

\section{Local cosmology: general relativity theory}

In the Newtonian theory for the local Universe (Sect. 2), the dark energy is treated as relativistic "fluid", in accordance with General Relativity, while the ordinary matter and space-time in which the matter move are described in terms of non-relativistic physics. Now we give a completely relativistic theory for the Local Universe.

\subsection{A spherically symmetric static metric}

It is most important that the set of the Newtonian spherically symmetrical equations (Eqs. (1)-(10) above) has an exact analogue in General Relativity. Indeed, there is a well-known GR solution for a point-like body on the vacuum background:

$\mathrm{d} s^{2}=F(r) \mathrm{d} t^{2}-r^{2} \mathrm{~d} \Omega^{2}-F(r)^{-1} \mathrm{~d} r^{2}$

where

$F(r)=1-2 G M / r-(r / A)^{2}$.

This time-independent metric describes a sphericallysymmetrical static space-time. Identifying the constant parameters $M$ and $A$ here with the mass of the Local Group and the vacuum time/length, respectively, we may use this metric 
for the local cosmology. The local flow particles move along geodesics in the space-time of Eqs. (12), (13).

In the limit of small deviations from the Newtonian (Galilean) space-time (quite appropriate for the Local Universe), the metric (Eq. (13)) takes the form:

$(F(r))^{\frac{1}{2}} \simeq 1-G M / r-\frac{1}{2}(r / A)^{2}=1+U(r)$,

where $U(r)$ is the gravitational potential of the Newtonian theory and exactly the same as in Eq. (5).

The metric of Eqs. (12), (13) representing the static GR model of the Local Universe contains all information about possible motions in this static space-time. In particular, the radial motions of the local flow particles are naturally the same (practically exactly) as in the Newtonian version (Eqs. (1)-(10)) of the model.

\subsection{Embedding the Local Universe into global space}

Furthermore, it is important that the $3 \mathrm{D}$ volume of the Local Universe with the metric of Eqs. (12)-(14) may be considered as "embedded" in the Friedmann global 3D cosmological space with the non-static metric

$\mathrm{d} s^{2}=\mathrm{d} t^{2}-a(t)\left[\mathrm{d} \Omega^{2}+\mathrm{d} \chi^{2}\right]$,

where $a(t)$ is the $3 \mathrm{D}$ curvature radius and/or the expansion scale factor (for $k=1$ ), which is proportional to the Friedmann radius $R(t, \chi)$ of Eq. (11).

Indeed, the Local Universe may be described as a spherical "vacuole" in the uniform distribution of matter, as given by the exact Einstein-Straus (1945) solution. The size of the vacuole $R_{\mathrm{L}}(t, M)$ increases with time in accordance with the Friedmann expansion solution $R_{\mathrm{L}} \propto a(t)$, and the central mass $M$ is equal to the mass of the (non-relativistic) matter within the sphere of radius $R_{\mathrm{L}}$ in the smooth matter distribution of the Friedmann Universe:

$M=\frac{4 \pi}{3} \rho(t) R_{\mathrm{L}}(t)^{3}=$ const.

In the vacuole model with the conjunction condition of Eq. (16), the environment of the Local Universe does not affect the dynamics inside it (cf. condition 1 assumed in Sect. 2). The Einstein-Straus (1945) solution demonstrates how the local nonFriedmann static cosmological model may be compatible with the Friedmann metric (and dynamics) of the global cosmological expansion.

It is easy to see that the zero-gravity surface has the radius $R_{V}=\frac{1}{2}\left(\rho_{\mathrm{M}} / \rho_{\mathrm{V}}\right)^{1 / 3} R_{\mathrm{L}}\left(t_{0}\right) \simeq\left(\frac{3}{14}\right)^{1 / 3} R_{\mathrm{L}}\left(t_{0}\right)$, where $R_{\mathrm{L}}\left(t_{0}\right)$ is the vacuole radius at present. The distance interval $(0.6-1) R_{\mathrm{L}}\left(t_{0}\right)$ is dominated by the vacuum in the Local Universe.

Arguing further along this line, one can imagine that the whole Universe may contain not one, but many (or an infinite number) of vacuoles of various sizes and masses. Moreover, a picture is theoretically possible in which vacuoles fill almost all cosmic space without intersecting each other. Such a complex non-uniform expanding structure is described exactly(!) by the equations above. Obviously in this picture the tiny contribution of uniform matter distribution between vacuoles can be neglected, and one obtains a highly non-uniform, but completely regular, cosmological model. The model describes the global expansion in terms of the relative motions of discrete masses that are in the centers of the vacuoles. The masses move apart from each other on the uniform vacuum background, in accordance with the Hubble law and the expansion factor given by the
Friedmann theory (Eq. (11)). In fact, because the zero-gravity radius is inside the vacuole in such a model, each vacuole expulses every other one and the expansion is generally accelerating (cf. Sect. 7.2 in Teerikorpi et al. 2005).

In this idealized model, the global Hubble constant, $H=$ $\dot{R} / R=\dot{a} / a$, is exactly the same for any two masses of the global expansion flow. In accordance with the Friedmann theory (Eq. (7)), it is presently $\left(t=t_{0}\right)$ :

$$
\begin{aligned}
H\left(t_{0}\right) \equiv H_{0} & =H_{\mathrm{V}}\left[1+\rho_{\mathrm{M}}\left(t_{0}\right) / \rho_{\mathrm{V}}\right]^{1 / 2} \simeq H_{\mathrm{V}}(1+3 / 7)^{1 / 2} \\
& \simeq 1.2 H_{\mathrm{V}} \simeq 72 \mathrm{~km} \mathrm{~s}^{-1} \mathrm{Mpc}^{-1}
\end{aligned}
$$

in good agreement (not surprisingly) with the concordance data (Spergel et al. 2002). It is also taken into account that $E=0$ in Eq. (11), according to the same data; $\rho_{\mathrm{M}}=3 \times 10^{-30} \mathrm{~g} / \mathrm{cm}^{3}$ is the concordance value for the mean matter (dark matter + baryons) density at present.

In terms of General Relativity, the asymptotic similarity of the local and global expansion flows is seen as clearly as in the Newtonian treatment of Sect. 3. Indeed, the static metric of Eqs. (12), (13) and the expanding metric of Eq. (15) have a common asymptotic in the limit when and where the vacuum antigravity dominates entirely. This limit is described by de Sitter's static solution. As is well-known, this solution has the metric of Eq. (12) with $F(r)=1-(r / A)^{2}$. The space-time of de Sitter's solution is determined by the vacuum alone, which is always static itself, and the vacuum completely controls both space-time metric and any geodesic in this space-time.

(Note that the vacuole model can be generalized: instead of the central mass, one may use a spherically symmetrical matter distribution of the same total mass, as in the Tolman-Bondi solution that is discussed e.g. by Gromov et al. (2001) in the context of the Local Universe.)

\section{The efficiency of vacuum cooling}

As demonstrated in Sects. 2, 3, the expansion flow described by the local cosmological model tends to a regular kinematical structure with the linear velocity-distance relation, $\dot{r}=r / A$, and the universal Hubble constant $H_{\mathrm{V}}=1 / A$, at the limit of the cosmic vacuum domination. It is important to emphasize that the regular self-similar parabolic regime is a generic asymptotic for all the expansion flow motions, independent of their initial conditions, in the Local Universe.

\subsection{From initial chaos to asymptotic order}

Indeed, in the model of Sects. 2, 3, the initial conditions are given in terms of the functions $\bar{E}(\chi)$ and $T(\chi)$. It is obvious from Eqs. (4), (9) that asymptotically both functions become negligible, since $E / r^{2} \rightarrow 0$ and $T / t \rightarrow 0$, in this limit. The self-similar motion described by Eq. (10) is a kind of dynamical attractor for a wide variety of motions possible in the gravity/antigravity potential of the Local Universe. If the energy condition of Eq. (6) is met, any particle escapes the interior of the zero-gravity sphere and its motion reaches the parabolic regime asymptotically (the universal Hubble constant). This basic evolutionary trend in the local flow drives it from initial chaos to asymptotic order (see also Chernin et al. 2004, 2005). It is also because this internal trend is so quick that the velocity dispersion of the local flow is rather low. To put this in a quantitative way, let us introduce a "random" velocity $v$ as a difference between the velocity $\dot{r}$ and 
a "regular" (asymptotic) velocity $r / A$. Then the velocity $v$ at the zero-gravity distance $r=R_{\mathrm{V}}$ is

$v\left(R_{\mathrm{V}}\right)=v_{1}=\left(R_{\mathrm{V}} / A\right)\left[\left(3+\bar{E} A^{2} / R_{\mathrm{V}}^{2}\right)^{1 / 2}-1\right]$.

When the trajectory of a body reaches a distance $r$ outside the zero-gravity surface, the random velocity $v(r)$ (for an arbitrary $\bar{E}$ ) is given by the relation:

$$
\begin{aligned}
v(r)= & \left(R_{\mathrm{V}} / A\right)\left[\left(r^{2} / R_{\mathrm{V}}^{2}+2+2 R_{\mathrm{V}} / r+2 v_{1} A / R_{\mathrm{V}}\right.\right. \\
& \left.\left.+v_{1}^{2} A^{2} / R_{\mathrm{V}}^{2}\right)^{1 / 2}-r / R_{\mathrm{V}}\right] .
\end{aligned}
$$

Note that different values of the total energy $E$ give an "initial" distribution of the velocity $v$. A comparison of $v(r)$ with $v_{1}$ enables us to see that the random velocity decreases along the trajectory and approaches zero asymptotically no matter what its initial value $v_{1}$ is.

\subsection{The vacuum cooling factor}

With the "vacuum cooling factor", $q_{\mathrm{V}} \equiv v_{1} / v$, we may measure the efficiency of the cooling mechanism:

$$
\begin{aligned}
q_{\mathrm{V}}= & \left(v_{1} A / R_{\mathrm{V}}\right)\left[\left(r^{2} / R_{\mathrm{V}}^{2}+2+2 R_{\mathrm{V}} / r+2 v_{1} A / R_{\mathrm{V}}\right.\right. \\
& \left.\left.+v_{1}^{2} A^{2} / R_{\mathrm{V}}^{2}\right)^{1 / 2}-r / R_{\mathrm{V}}\right]^{-1} .
\end{aligned}
$$

In the simplest case of the parabolic motion $(\bar{E}=0)$, the velocity $v_{1}=(\sqrt{3}-1) R_{\mathrm{V}} / A$, and we have:

$$
v(r)=\left(R_{\mathrm{V}} / A\right)\left[\left(r^{2} / R_{\mathrm{V}}^{2}+2 R_{\mathrm{V}} / r\right)^{1 / 2}-r / R_{V}\right] .
$$

Then the cooling factor in the parabolic expansion flow is

$q_{\mathrm{V}}(r) \equiv v_{1} / v(r)=(\sqrt{3}-1)\left[\left(r^{2} / R_{\mathrm{V}}^{2}+2\right)^{1 / 2}-r / R_{\mathrm{V}}\right]^{-1}$.

One can see that the random velocity is diminished by a factor 3 during the time when the body covers the path from $r=R_{\mathrm{V}}$ to the distance $r \simeq 2 R_{\mathrm{V}} \simeq 3 \mathrm{Mpc}$. When the same body reaches, say, distances of 4 or $6 R_{\mathrm{V}}$, the cooling factor increases to $q_{\mathrm{V}}=12$ and $q_{\mathrm{V}}=56$, correspondingly.

Now we compare the vacuum cooling with the usual cosmological adiabatic cooling. The latter is described by the relation $v a(t)=$ constant, which only gives the factors 2,4 , and 6 when the scale factor $R(t)$ increases 2, 4, or 6 times; this is considerably less than the corresponding factors 3,12 , and 56 in the expansion flow of our model.

It is also interesting to follow - for a contrast - the dynamics of the same expansion, but in the absence of a vacuum. With the same statement of the problem as above, but with $\rho_{\mathrm{V}}=0$, we would have:

$\dot{r}^{2}=2 G M / r+2 \bar{E}$.

When, for instance, $\bar{E}>0$, the asymptotic (as $r$ goes to infinity) motion is inertial and the velocity-distance law is a linear one: $\dot{r}=r / t$, where the "Hubble factor", $H=1 / t$, depends only on time. This means that the flow may cool down without the vacuum just because of its expansion. But the efficiency is then much lower. Indeed, taking $r / t$ as a regular expansion velocity, we may introduce the random velocity $v$ by the relation $\dot{r}=r / t+v$. Then

$v=(2 E)^{1 / 2}\left[\left(1+\frac{G M}{2 E r}\right)^{1 / 2}-1\right]$.

In the first approximation for $v /(2 E)^{1 / 2}<1$, this leads to the "adiabatic" relation $v r=$ constant. As a result, the cooling factor in the absence of vacuum, $q \propto r$, is significantly smaller than the vacuum cooling factor $q_{\mathrm{V}}$ above.

Thus, vacuum cooling is very efficient and results in a dynamical evolution of the outflow from chaos to order. When applied to the nearest distances 1-3 Mpc, the non-Friedmann cosmology (for a parabolic expansion) gives a typical velocity dispersion between $v_{1}=(\sqrt{3}-1) R_{\mathrm{V}} / A=60 \mathrm{~km} \mathrm{~s}^{-1}$ at $r=R_{\mathrm{V}}$ and $v\left(r=2 R_{\mathrm{V}}\right)=\frac{1}{3} v_{1}=20 \mathrm{~km} \mathrm{~s}^{-1}$ at $r=2 R_{\mathrm{V}}$, which is near the observed figure 30-60 $\mathrm{km} \mathrm{s}^{-1}$ (Karachentsev \& Makarov 2001; Karachentsev et al. 2002, 2003a; Ekholm et al. 2001; Paturel \& Teerikorpi 2005).

\section{Significance of the universal Hubble constant}

In this and the next sections, we discuss new observational prospects offered by the local cosmology. The background is naturally the global cosmology where both $H_{\mathrm{V}}$ and $H_{0}$ are among the basic parameters. As we show here, there are good prospects for the uniform vacuum density and the universal $H_{\mathrm{V}}$ to be determined locally, but the exact value of the Hubble constant $H_{0}$ refers to the average global expansion rate that cannot be measured in a local, inhomogeneous environment. Locally, a good advantage is the high, $10 \%$ or better, accuracy of distance measurements, while distances continue to be hampered by low accuracy and systematic errors on large scales.

\subsection{The stochastic Hubble expansion rate}

Of course, a "precision cosmology" value of $H_{0}$ may be obtained from analysis of the CMBR. However, this still depends on assumed cosmological physics and on dark-matter and darkenergy components (Spergel et al. 2003), and it would be very important to have an independent direct measurement on large scales. Deep-space measurements of $H_{0}$, even bypassing local calibrations, from the Sunuyaev-Zeldovich effect and the time delay in gravitational lens images, are still affected by rather large systematic errors. The same must be said about the proposed way of studying the distance scale via the Eddington efficiency of luminous quasars (Teerikorpi 2005).

In intermediate and closer space, the HST Key Project (Freedman et al. 2001) derived $H_{0}=72 \pm 8 \mathrm{~km} \mathrm{~s}^{-1} \mathrm{Mpc}^{-1}$, using secondary distance indicators calibrated from local galaxies whose distances are known from Cepheids. This value agrees with the value from the CMBR analysis and is a part of the concordance picture. However, one should not lose sight of the fact that $H_{0}$ has not yet been determined directly on large scales with a high accuracy and there are still possible sources of systematic error in the HST value for $H_{0}$ (Paturel \& Teerikorpi 2005; Sandage et al. 2006).

Furthermore we wish to emphasize that the measured Hubble constant is not the same between all pairs of galaxies or clusters - contrary to the simple vacuole model. The expansion rate varies depending on the matter and gravity field variations in the space between the observer and the object. Therefore the really measured Hubble parameter is instead a stochastic variable that can be expressed relative to the truly universal vacuum Hubble constant $H_{\mathrm{V}}$ and the global time-dependent average Hubble constant $H_{0}=H_{\mathrm{V}}\left(1+\frac{\rho_{\mathrm{M}}\left(t_{0}\right)}{\rho_{\mathrm{V}}}\right)$. In any particular measurement

$H_{i}=H_{\mathrm{V}}\left(1+\frac{\rho_{M}\left(t_{0}\right)}{\rho_{\mathrm{V}}}\right)^{1 / 2}\left(1+\Delta_{i}\right) \mathrm{km} \mathrm{s}^{-1} \mathrm{Mpc}^{-1}$.

The deviation $\Delta_{i}$ must depend on the spatial scale of measurement, related to the average matter density contrast. 


\subsection{The local density contrast}

The situation at small distances is also not the ideal one described by the vacuole model; e.g., the conjunction condition of Eq. (16) may not be fulfilled in the Local Universe, unless we consider a small volume around the Local Group. This is because the mass of the Local Group is considerably less than the mass of a uniform sphere with the cosmological density $\rho_{M}\left(t_{0}\right)$ and the radius 5-10 Mpc. In fact, a cosmological volume containing the non-relativistic mass $M$ of the Local group (dwarf galaxies contribute negligibly within a few percent accuracy) has the present-day radius

$R(M)=\left[M /\left(\frac{4 \pi}{3} \rho_{\mathrm{M}}\right)\right]^{1 / 3}=R_{\mathrm{V}}\left(2 \frac{\rho_{\mathrm{V}}}{\rho_{\mathrm{M}}}\right)^{1 / 3} \simeq 2.2 \mathrm{Mpc}$.

As this is below typical distances between galaxy groups locally, there may be a density deficit. This would not influence the validity of the very local model that only depends on the mass of the Local Group and the value of the vacuum density, but it would naturally change the expected value of the expansion rate of the Local Universe.

On the other hand, if we allow for an extended distribution of dark matter beyond the $1 \mathrm{Mpc}$ radius of the Local Group, perhaps following a fractal density law (e.g. Teerikorpi et al. 1998; Baryshev \& Teerikorpi 2002, 2006), then with the fractal dimension $D=1.2$ or the correlation exponent $\gamma=1.8$, the cosmological density would be reached around 3.2 Mpc. With $D=2$, this distance would be $8 \mathrm{Mpc}$. We do not know the exact distribution of dark matter, but clearly it could add to the total mass so that the conjunction condition is locally fulfilled.

Inspection of Fig. 4 in Karachentsev et al. (2003b) shows that within about $3 \mathrm{Mpc}$ the Local Group is alone, while there are six other groups within $6 \mathrm{Mpc}$. This would roughly correspond to an average matter density of $0.3 \rho_{M}$ within $3 \mathrm{Mpc}$, if there is no dark matter related to the Local Group beyond $1 \mathrm{Mpc}$, while the average matter density would be close to $\rho_{\mathrm{M}}$ with the dark-matter distribution with $\gamma=1.8$. Hence, it seems that locally the average matter density could be roughly in the range $(0.3-1) \rho_{\mathrm{M}}$, depending on the distribution of the dark matter around the groups. This would agree with other evidence that within a distance of $8 \mathrm{Mpc}$ there is only a small density excess, if any (Hudson 1992; Macció et al. 2005). We note that generally a randomly placed observer would be expected to be surrounded by a clear density excess (cf. Sect. 8 in Teerikorpi et al. 2005).

\subsection{The local expansion rate and $H_{V}$}

Because of the relatively low matter content around us, the domination of cosmic vacuum may be stronger in the Local Universe than on average over the Universe as a whole in the present epoch. This special feature of the Local Universe could offer better chances for vacuum measurements there.

First, if there is a density deficit, the local Hubble constant $\bar{H}$ is in general less than the global constant $H_{0}$ and closer here to the universal Hubble constant $H_{\mathrm{V}}$, at least on sufficiently long scales: $H_{\mathrm{V}}<\bar{H}_{0}<H_{0}$. Secondly, dwarf galaxies close to the Local Group may be sensitive indicators of the vacuum, especially if the LG mass is concentrated within a small volume (the local cosmology model; Sects. 2, 3). We discuss this in the next section.

To illustrate these considerations, we take a look at Eqs. (1)-(10) of the local cosmology. If one assumes that the local flow contains mostly parabolic or nearly parabolic trajectories, then the very local Hubble rate is

$\bar{H}_{0}(r)=\frac{\dot{r}}{r}=H_{\mathrm{V}}\left(1+\frac{2 G M A^{2}}{r^{3}}\right)^{1 / 2}=H_{\mathrm{V}}\left(1+2\left(\frac{R_{\mathrm{V}}}{r^{3}}\right)^{3}\right)^{1 / 2}$

When $2 G M A^{2} / r^{3}=2\left(R_{\mathrm{V}} / r\right)^{3}$ is small, the flow is close to the asymptotic regime, and the deviation of $\bar{H}_{0}$ from $H_{\mathrm{V}}$ is less than, say, $6 \%$ at distances $r \gtrsim 2.5 R_{\mathrm{V}} \simeq 3-4 \mathrm{Mpc}$.

Of course, there are distortions of the local flow caused by the Virgo cluster and other environmental effects, but these may still be relatively weak at $r<3-4 \mathrm{Mpc}$. This is supported by various observational studies of the local Hubble flow, as summarized in Teerikorpi et al. (2005). In these studies, the local Hubble constant is found within the interval 56-70 $\mathrm{km} \mathrm{s}^{-1} \mathrm{Mpc}^{-1}$, so that the central value here is indeed quite close to the theoretically expected value of $H_{\mathrm{V}}$.

\section{The shortest-distance indicators of dark energy}

The theory of the local flow (Sects. 2, 3) leads to two clear predictions that may be observationally tested:

(1) There is a lower limit for the flow velocities outside the zerogravity sphere. Indeed, the escaped galaxies have the energy $\bar{E} \geq U_{\max }=-\frac{3}{2} G M / R_{\mathrm{V}}$, as seen from Eq. (6). For any distance $r$, this inequality implies that the flow velocity must be large enough:

$\dot{r}(r) \geq V_{0}(r) \equiv H_{\mathrm{V}} R_{\mathrm{V}}\left(2 R_{\mathrm{V}} / r+r^{2} / A^{2}-3\right)^{1 / 2}$.

(2) There is a lower limit for the distances in the local Hubble flow: $r \geq R_{\mathrm{V}}$.

Prediction (1) is critical: if only a single isolated and accurately observed galaxy is found to violate this limitation, the theory or the values of its parameters must be rejected or modified. Prediction (2) is closely related to the observed kinematical structure of the flow and may serve for effective measurements of dark-energy density.

\subsection{Comparison with observational data}

Here we concentrate on the nearest distances that are most sensitive to the test. It is also here where the distances (TRGB, Cepheids) are expected to be less influenced by selection effects that cause systematic errors (Teerikorpi 1997; Paturel \& Teerikorpi 2005).

Indeed, recent data on the Local group vicinity (1-3 Mpc from the group barycenter) are given by Karachentsev et al. (2002, 2004). In the critical distance interval 1-2 Mpc, 8 galaxies were studied. Their distances were measured with a typical reported accuracy of $10 \%$ and the accuracy of the velocities is $1-2 \mathrm{~km} \mathrm{~s}^{-1}$. The galaxy SagDIG is the nearest to the Local group barycenter with a distance of $1.2 \mathrm{Mpc}$. Its velocity, $23 \mathrm{~km} \mathrm{~s}^{-1}$ relative to the group barycenter, is the lowest in the flow. The next closest galaxy is SexB with the distance of $1.6 \mathrm{Mpc}$ and velocity of $111 \mathrm{~km} \mathrm{~s}^{-1}$. The three galaxies, Antlia, NGC 3109, and SexA, have almost equal distances near 1.7 Mpc and the velocities of 66,110 , and $94 \mathrm{~km} \mathrm{~s}^{-1}$, respectively. The galaxy KKR25 has a distance $1.8 \mathrm{Mpc}$ and a velocity $68 \mathrm{~km} \mathrm{~s}^{-1}$. The galaxies E294-010 and KKH98 are located at a distance near $2 \mathrm{Mpc}$, and they have the velocities 81 and $152 \mathrm{~km} \mathrm{~s}^{-1}$. We also add NGC 300, with its Cepheid distance $(1.93 \mathrm{Mpc})$ recently measured by Gieren et al. (2004), and its $125 \mathrm{~km} \mathrm{~s}^{-1}$ velocity 


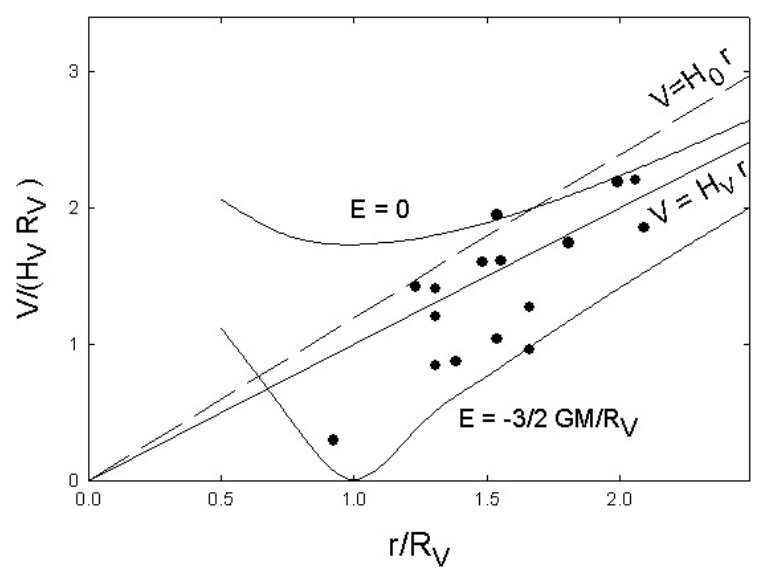

Fig. 1. The Hubble diagram for very nearby galaxies, with the units $R_{\mathrm{V}}=1.3 \mathrm{Mpc}$ and $H_{\mathrm{V}} R_{\mathrm{V}}=78 \mathrm{~km} \mathrm{~s}^{-1}$ used for $x$ - and $y$ - axis, respectively. The linear velocity-distance relation is shown by two straight lines, one for the universal Hubble constant $H_{\mathrm{V}}$ and the other for the global Hubble constant $H_{0}$. The trajectories for the parabolic motion $(E=0)$ and for the lower limit velocity $V_{0}$ are given.

and seven other galaxies with $r \leq 2.5 \mathrm{Mpc}$ from the catalogue of Karachentsev et al. (2004). Thus we have all the galaxies with known distances (from the TRGB or Cepheid method) between $1 \mathrm{Mpc}$ and $2.5 \mathrm{Mpc}$.

All 16 galaxies with their barycentric distances from the LG are plotted on the Hubble diagram in Fig. 1, where the units $R_{\mathrm{V}}=1.3 \mathrm{Mpc}$ and $H_{\mathrm{V}} R_{\mathrm{V}}=78 \mathrm{Mpc}$ have been used for the $x$ - and $y$-axes, respectively. The linear velocity-distance relation is shown by the two straight lines, one for the universal Hubble constant $H_{\mathrm{V}}$ and the other for the global Hubble constant $H_{0}$. The trajectories for the parabolic motion $(E=0)$ and the lower limit velocity $V_{0}$ are also given. We can see in Fig. 1 that all the galaxies here obey the restriction of Eq. (28).

We also see that - in agreement with the discussion of Sects. 2, 3 - the two curves reveal a trend to converge to the universal (with $H_{\mathrm{V}}$ ) linear velocity-distance relation. The lower limit velocity approaches the relation rather closely at the distances 5-7 Mpc (mentioned also in the section above), which could provide another sensitive interval for an observational test.

\subsection{Towards weighing dark energy}

In global cosmology, the dark energy of the cosmic vacuum has shown itself in observation (Riess et al. 1988; Perlmutter et al. 1999) at very large distances that are comparable to the vacuum length $A$ or the horizon radius. The corresponding redshifts are near

$1+z_{\mathrm{V}}=\left[2 \rho_{\mathrm{V}} / \rho_{\mathrm{M}}\left(t_{0}\right)\right]^{1 / 3} \simeq 1.7$

At $z=z_{\mathrm{V}} \sim 0.7$, the global cosmological expansion transits from deceleration to acceleration.

The redshift $z_{\mathrm{V}}$ is a clear global counterpart of the local zerogravity radius $R_{\mathrm{V}}$ : the local flow transits from deceleration to acceleration at $r=R_{\mathrm{V}}$. The difference between them is, however, essential. In global cosmology, the balance of gravity and antigravity occurs only for one moment of time (7-8 Gyr ago) and simultaneously for the whole Universe. In contrast, in local cosmology, the balance exists for $\sim 10 \mathrm{Gyr}$, but only at the zero-gravity surface. In a sense, what is temporal in the global cosmology is spatial in the local one, and vice versa.

Could observations at short distances near $R_{\mathrm{V}}=1-2 \mathrm{Mpc}$ be as useful and important as the observations at high redshifts near $z_{\mathrm{V}} \sim 1$ ? The theory prediction (2) above indicates that the distance $R_{\mathrm{V}}$ plays a key role in the dynamics of the local flow. As first mentioned in Paper I, the local Hubble flow starts very close to the zero-gravity sphere, if not exactly at $r=R_{\mathrm{V}}$. This is hardly a funny numerical coincidence with no physical sense. Instead, the coincidence is quite suggestive, because it points out the shortest distances where the dark energy may be detected and measured from its effect on velocities.

Indeed, as seen from Fig. 1 (and from the basic equations of the theory as well), the velocities of the flow trajectories have minima at $r=R_{\mathrm{V}}$. The location of the minima is the same for all the trajectories, independent of their initial conditions. If the location of the velocity minimum $r=R_{\mathrm{V}}$ is found from observations, the universal Hubble constant may be directly determined (following from the definition of Eq. (3)):

$$
H_{\mathrm{V}}=60\left(\frac{M}{1.5 \times 10^{12} M_{\odot}}\right)^{1 / 2}\left(\frac{1.3 \mathrm{Mpc}}{R_{\mathrm{V}}}\right)^{3 / 2} \mathrm{~km} \mathrm{~s}^{-1} \mathrm{Mpc}^{-1}
$$

Similarly, the density of the vacuum dark energy is

$$
\rho_{\mathrm{V}}=7 \times 10^{-30}\left(\frac{M}{1.5 \times 10^{12} M_{\odot}}\right)\left(\frac{1.3 \mathrm{Mpc}}{R_{\mathrm{V}}}\right)^{3} \mathrm{~g} / \mathrm{cm}^{3} .
$$

Now we turn to the data of Fig. 1 again and inspect them for the velocity minimum location. Noting that the velocities appear to still be decreasing around $r / 1.3 \mathrm{Mpc} \approx 1.4$ a robust conservative estimate follows for the upper limit value of the zero-gravity radius:

$R_{\mathrm{V}} \lesssim 1.8 \mathrm{Mpc}$.

This agrees with the theoretically expected provisional value $1.3 \mathrm{Mpc}$ that we had in mind above. Combined with Eqs. (30), (31), this leads to observational restrictions on the values $H_{\mathrm{V}}^{2} / M$ and $\rho_{\mathrm{V}} / M$. Assuming $M=1.5 \times 10^{12} M_{\odot}$, one has from Eq. (32) an independent estimate for the lower limit of the dark energy density in the Local Universe:

$\rho_{\mathrm{V}} \gtrsim 3 \times 10^{-30} \mathrm{~g} / \mathrm{cm}^{3}$.

If the lowest velocity in the flow (that of the galaxy SagDIG) is tentatively identified as showing the location of the velocity minimum in the flow, we may get $R_{\mathrm{V}}=1.2 \pm 0.1 \mathrm{Mpc}$. With this (not so reliable) estimate

$\rho_{\mathrm{V}}=(9 \pm 2) \times 10^{-30} \mathrm{~g} / \mathrm{cm}^{3}$.

In these estimates we have fixed the mass of the Local Group to $1.5 \times 10^{12} M_{\odot}$. This value corresponds roughly to the best-fit value of the extended halo of M $31\left(0.7 \times 10^{12} M_{\odot}\right.$ by Evans et al. 2000) plus the mass of the Milky Way, taken to be equal or somewhat lower than that of M 31 (e.g. Karachentsev \& Kashibadze 2005 give the ratio 0.8 ). As there is still considerable uncertainty in direct measurements of these local masses (cf. Wilkinson \& Evans (1999) on the Milky Way), the above estimates of $\rho_{V}$ may be viewed as normalized to the adopted value of the Local Group mass $\left(\rho_{\mathrm{V}} \propto M\right)$ or, alternatively, it is interesting to write them in terms of the mass-to-luminosity ratio of the Local Group. Van den Bergh (1999) gives (in the $V$ band) $M / L=44$ for $M=2.3 \times 10^{12} M_{\odot}($ corresponding to 29 for our lower $M)$, so we may write e.g. the first estimate as

$\rho_{\mathrm{V}} \gtrsim 3 \times 10^{-30}\left[(M / L)_{\mathrm{LG}} / 29\right] \mathrm{g} / \mathrm{cm}^{3}$.

We give these values as examples of what could be done with higher precision using considerably more numerous distance 
determinations in the interval 1-2.5 Mpc. One expects many more still unmeasured dwarf galaxies within these distances (Karachentsev et al. 2004). In any case, the very initial local flow with the shortest distances and lowest redshifts - in drastic contrast to the important, but very difficult high redshift SN studies - have proved to be a sensitive indicator and "weighing device" of the dark energy.

\section{Discussion}

The current standard cosmological model may actually be seen as containing two fundamental and equally important sectors. The traditional global cosmology studies the uniform and evolving space-time on the large spatial scales of 300-1000 Mpc or more, while local cosmology deals with the non-uniform static space-times of the Local Universe and many (or infinite number) of the other more or less similar local regions. The global sector uses the Friedmann solution as the theoretical basis, while the local one needs a non-Friedmann theory that we proposed and described above (Sects. 2, 3) in a simple analytical version. We also explained (Sect. 3) how the two sectors of the whole model might be linked to each other, at least, in our example of an ideal vacuole scheme.

The omnipresent and perfectly uniform dark energy of the cosmic vacuum is the major physical factor that unifies the two sectors of the model. It is especially important that, in the present-day Universe, the vacuum with its antigravity dominates dynamically on global scales and also within individual local volumes outside the zero-gravity surfaces. On the global scales, the vacuum antigravity accelerates the general cosmological expansion produced by the initial Big Bang. Locally, the vacuum antigravity makes dwarf galaxies move from the Local Group and separate from each other with acceleration, finally establishing the Hubble flow.

On both global and local scales, the vacuum provides the motions with a common asymptotic behavior that is the selfsimilar parabolic expansion entirely controlled by the vacuum antigravity. This dynamical regime is an attractor for a wide range of real motions on the cosmic vacuum background, including the Friedmann uniform expansion and the local spatially non-uniform flow. In this limit, the expansion follows the exponential law, both globally and locally: $r \propto R \propto \exp (t / A)$. As a result, the linear velocity-distance relation establishes, $\dot{r} \propto$ $r ; \dot{R} \propto R$, i.e. the Hubble expansion law. Both locally and globally, the expansion rate is given by the same universal physical constant related directly to the vacuum density, in the limit: $H_{\mathrm{V}}=\dot{r} / r=\dot{R} / R=1 / A \simeq 60 \mathrm{~km} \mathrm{~s}^{-1} \mathrm{Mpc}^{-1}$. Correspondingly, the maximally symmetric space-time described by the de Sitter metric serves as the universal asymptotic for both Friedmann global non-static space-time and the non-Friedmann local static space-time.

The concordance data for global cosmology and the data for the Local Universe indicate that the present-day states of both global and local expansion flows are not far from the universal asymptotic regime. It is because of this that the observed local expansion rate is so close to the global Hubble constant (which has been rightly considered as a big cosmic puzzle - see Sandage 1999). This fact also explains why the velocity dispersion is rather low in the local expansion flow (which has also been a true mystery).

The simplest analytical version of the local model described in this paper is supported by computer simulations (Chernin et al. 2004, 2005) that extend the model into the interior of the zero-gravity surface. The simulations describe the Local Group as a binary (the Milky Way and M 31) galaxy, in accordance with the classical Kahn-Woltjer (1959) model. They confirm that the differences from the point-mass analytical model are indeed small outside the zero-gravity surface. The binary produces relatively small deviations from spherical symmetry in the local gravity field at $r \geq R_{\mathrm{V}}$ and also a relatively weak time dependence of the gravity field at these distances. In particular, the simulations show that the zero-gravity surface is almost perfectly spherical at present.

The simulations describe all the history of the local flow during 12-13 Gyr since the formation of the Local Group. They also enable us to study the "initial conditions" for the local flow, and indicate that the Little Bang model (Byrd et al. 1994) for the initial chaotic state of the Local Group (see also van den Bergh 2003) could produce a flow of galaxies across the zero-gravity surface, and their radial velocities are later amplified by the antigravity push of the cosmic vacuum outside the zero-gravity surface.

Our analytical theory and simulations agree closely with the large $N$-body $\Lambda$ CDM cosmological simulations (Ostriker \& Suto 1990; Suto et al. 1992; Governato et al. 1997; Nagamine et al. 1999, 2000; Macciò et al. 2005). These simulations give important insights into the early history of the Local Universe when its matter started to separate from the general cosmological expansion at the cosmic age of $0.5-1.5$ Gyr. The picture is complementary to ours and demonstrates that the Local Universe with a massive group in its center and the quiescent expansion outflow is fairly typical for scales of a few megaparsecs. Such local structures emerge in a natural way from the standard picture of gravitational instability with the Harrison-Zeldovich initial spectrum.

Very important is that these "global" simulations have shown - confirming the original approach of our Paper I - that the dark energy is necessary for explaining cool local flows (Macciò et al. 2005).

\section{Conclusions}

We summarize the main results of the present study:

- A non-Friedmann model with a non-uniform, static spacetime was proposed for the neighborhood of the Local Group in order to describe the trajectories of galaxies flowing across the local zero-gravity surface at $r=R_{\mathrm{V}} \simeq 1.3 \mathrm{Mpc}$. The model is given both within Newtonian theory and GR.

- It was shown that this local space-time, when treated within GR, may be embedded in the global Friedmann space-time, and the Local Universe may described as a spherical vacuole, according to the Einstein-Straus solution.

- We emphasize the difference between the truly universal Hubble constant $H_{\mathrm{V}}$, depending only on the constant vacuum density, and the measured local or global Hubble constant $H_{0}$ that depend both on spatial matter distribution and scale and on the cosmic time.

- We have inspected the predicted trajectories of the outflowing galaxies and demonstrated analytically the efficiency of vacuum cooling, which is higher than the usual adiabatic cooling: all trajectories within the vacuole tend towards the Hubble relation with the universal vacuum Hubble constant $H_{\mathrm{V}}$. This gives a natural explanation for both the low velocity dispersion in the flow and its closeness to the global Hubble constant.

- The possible absence of turning-down of the velocitydistance relation close to the Local Group (Ekholm et al. 2001; Thim et al. 2003) and, in general, the absence of 
the infall towards local groups (Whiting 2005) may not be a problem for the present model, where the outflowing dwarfs remain close to the universal Hubble relation and have a positive minimum velocity at $r=R_{V}$.

- An interesting result is that all out-flowing trajectories have a velocity lower limit at any distance, corresponding to the total energy just required to reach $r=R_{\mathrm{V}}$.

- Preliminary comparison with available distance and velocity data for nearby galaxies, 1-2 Mpc, shows agreement of the predicted trajectories with $E \leq 0$ and may suggest the expected minimum around 1.3 Mpc. The local Hubble diagram $V /\left(H_{\mathrm{V}} R_{\mathrm{V}}\right)$ vs. $r / R_{\mathrm{V}}$ is a promising instrument for such studies.

Finally, the local Hubble flow is not all that mysterious any more, but it may instead be seen now as a natural tool for detecting and studying the vacuum antigravity and dark energy. In fact, we have already used it for these purposes since our Paper I. Combining our theory and computer simulations with precision observations, we have discovered dark energy in the Local Universe.

- The flow provides strong evidence of the presence of dark energy in the Local Universe on the small scales down to $\sim 1 \mathrm{Mpc}$; the local DE density seems to be close to that measured on the largest cosmological scales.

- The dark energy in the Local Universe can be well described in the simplest way as the cosmological constant or cosmic vacuum with the density-to-pressure ratio equal to -1 ; this agrees with the concordance global cosmological model.

- The dark energy with these properties might have existed unchanged for 12-13 Gyr in the Local Universe, since the formation of the Local Group. Global cosmology has no data on the dark energy at such distances/times.

These conclusions need and deserve further observational verification. We advocate extensive systematic high-precision observations of galaxies, focused on short distances, especially 0.5-2 Mpc. The advantages of such data are clear. These observations may give us increasingly exact information about dark energy. In particular, the local measurement of the dark energy density can be much improved as compared with our preliminary estimate here. Strong constraints may also be obtained on (1) deviations from the standard density-to-pressure ratio in the present-day Local Universe, (2) the time variation of the dark matter density for a period of 12-13 Gyr since the formation of the Local Group, and (3) the time variation of the densityto-pressure ratio during the same long cosmological time. Such data could also give empirical input for studies of how darkenergy properties and their time dependence influence the evolution of overdensities in small-scale non-linear regimes (Mota $\&$ van de Bruck 2004).

Observations near the zero-gravity surface of the radius $R_{\mathrm{V}} \sim$ $1 \mathrm{Mpc}$ may prove to be as useful as the observations near the redshift $z_{\mathrm{V}} \sim 1$ of the zero-gravity moment in the global Universe. With increasingly precise measurements of distances and velocities in the Local Universe, the local sector will be a central arena of further progress in cosmology.

Acknowledgements. We appreciate valuable discussions with Yury Efremov, Mauri Valtonen, and our other colleagues at Sternberg Institute and Tuorla Observatory. We thank the referee for useful comments.

\section{References}

Baryshev, Yu., \& Teerikorpi, P. 2002, Discovery of Cosmic Fractals (Singapore: World Scientific)

Baryshev, Yu., \& Teerikorpi, P. 2006, Bull. Spec. Astrophys. Obs., 59, 92

Baryshev, Yu., Chernin, A., \& Teerikorpi, P. 2001, A\&A, 378, 729

Chernin, A. D. 2001, Physics-Uspekhi, 44, 1099

Chernin, A. D. 2005 [arXiv: astro-ph/0503358]

Chernin, A. D., Teerikorpi, P., \& Baryshev, Yu. V. 2000, Adv. Space Res., 31, 459 [arXiv: astro-ph/0012021] - Paper I

Chernin, A. D., Karachentsev, I. D., Valtonen, M. J., et al. 2004, A\&A, 415, 19

Chernin, A. D., Karachentsev, I. D., Valtonen, M. J., et al. 2005 [arXiv:astro-ph/0507364]

Dolgachev, V. P., Domozhilova, L. M., \& Chernin, A. D. 2003, Astr. Rep., 47, 728

Ekholm, T., Baryshev, Yu., Teerikorpi, P., Hanski, M., \& Paturel, G. 2001, A\&A, 368, L17

Einstein, A., \& Straus, E. G. 1945, Rev. Mod. Phys., 17, 120

Hudson, M. J. 1993, MNRAS, 265, 43

Freedman, W. L., Madore, B. F., Gibson, B. K., et al. 2001, ApJ, 553, 47

Governato, F., Moore, B., Cen, R., et al. 1997, New Astron., 2, 91

Gromov, A., Baryshev, Yu., Suson, D., \& Teerikorpi, P. 2001, Gravitation \& Cosmology, 7, 140

Kahn, F. D., \& Woltjer, L. 1959, ApJ, 130, 705

Karachentsev, I. D., \& Kashibadze, O. G. 2005 [arXiv:astro-ph/0509207]

Karachentsev, I. D., \& Makarov, D. I. 1996, AJ, 111, 794

Karachentsev, I. D., \& Makarov, D. I. 2001, Astrofizika, 44, 5

Karachentsev, I. D., Sharina, M. E., Makarov, D. I., et al. 2002, A\&A, 389, 812

Karachentsev, I. D., Makarov, D. I., Sharina, M. E., et al. 2003a, A\&A, 398, 479

Karachentsev, I. D., Chernin, A. D., \& Teerikorpi, P. 2003b, Astrofizika, 46, 491

Karachentsev, I. D., Karachentseva, V. E., Huchtmeier, W. K., \& Makarov, D. I. 2004, AJ, 127, 2031

Karachentsev, I. D., Dolphin, A. E., \& Tully, R. B. 2005 [arXiv: astro-ph/0511648]

Macciò, A. V., Governato, F. \& Horellou, C. 2005, MNRAS, 359, 941

Mota, D. F., \& van de Bruck, C. 2004, A\&A, 421, 71

Nagamine, K., Cen, R., \& Ostriker, J. P. 1999, Bul. Amer. Astron. Soc., 31, 1393

Nagamine, K., Ostriker, J. P., \& Cen, R. 2001, ApJ, 553, 513

Ostriker, J. P., \& Suto, Y. 1990, ApJ, 348, 378

Paturel, G., \& Teerikorpi, P. 2005, A\&A, 443, 883

Perlmuter, S., Aldering, G., Goldhaber, G., et al. 1999, ApJ, 517, 565

Rekola, R., Richer, M. G., McCall, M. L., et al. 2005, MNRAS, 361, 330

Riess, A. G., Filippenko, A. V., Challis, P., et al. 1998, AJ, 116, 1009

Sandage, A. 1986, ApJ, 307, 1

Sandage, A. 1999, ApJ, 527, 479

Sandage, A., Tammann, G. A., Saha, A., et al. 2006, ApJ, submitted [arXiv: astro-ph/0603647]

Spergel, D. N., Verde, L., Peiris, H. V., et al. 2003, ApJS, 148, 175

Strauss, M. A., Cen, R., \& Ostriker, J. P. 1993, ApJ, 408, 389

Suto, Y., Cen, R., \& Ostriker, J. P. 1992, ApJ, 395, 1

Teerikorpi, P. 1997, ARA\&A, 35, 101

Teerikorpi, P. 2005 [arXiv: astro-ph/0510382]

Teerikorpi, P., Hanski, M., Theureau, G., et al. 1998, A\&A, 334, 395

Teerikorpi, P., Chernin, A., \& Baryshev, Yu. 2005, A\&A, 440, 791

Thim, F., Tammann, G., Saha, A., et al. 2003, ApJ, 590, 256

van den Bergh, S. 1999, A\&A Rev., 9, 273

Whiting, B. A. 2003, ApJ, 587, 186

Whiting, B. A. 2005, ApJ, 622, 217

Wilkinson, M. I., \& Evans, N. W. 1999, MNRAS, 310, 645 